\title{
Clamping system for series connected IGBTs to avoid transient break down voltages
}

\author{
Masoomeh Boloorian, Tonny W.Rasmussen, Walid Z. El-Khatib \\ Technical University of Denmark \\ Elektrovej 325 \\ 2800 Lyngby \\ Denmark
}

\begin{abstract}
IGBTs (Insulated Gate Bipolar Transistor) can be used for power DC-DC converters at higher voltages. A series connection is needed due to the blocking capability limit of $6500 \mathrm{~V}$. The voltage must be shared among the IGBTs both dynamic when switching and blocking mode. A non-synchronized switching creates transient voltage that might damage the IGBT. The non-synchronized switching comes from different delays in the gate drivers and deviation in the IGBT parameters. This paper investigates in theory and practice a solution contains of clamp circuit, dynamic and static circuit. Turning on process of the stack of three IGBTs are in focus in a buck converter design. An operating voltage closer to the IGBT limit is used in order to press the system. For the simulations (Pspice) and experimental tests, fast IGBTs (1200V /30A IXYH40N120C3) are used to show the good dynamic of the chosen solution. The Lab tests also show that because of the deviation in parameters pre adjustment of the gate signals is needed. The experimental tests have been carried out in different clamp voltage to finally have clamp voltage close to the blocking voltage of the IGBTs and maximum 2A as output current. Examples of wave forms for voltages are given and discussed.
\end{abstract}

\section{Introduction}

Series connection of the IGBTs is used to avoid costly and complicated methods such as using transformer and multilevel topologies to increase the voltage rate in the converters [1]. A circuit should protect the string of IGBTs against overvoltage and ensure voltage sharing between IGBTs. Active gate drivers [2] and [3] can be a solution. In this paper, the method in [4] is used to investigate for three high speed and high voltage IGBTs in series. In contrary with the paper [4] three fast IGBTs with clamp voltage close to the blocking voltage of the IGBTs are tested. Dynamic and static sub circuits contain of RCD and R, respectively, are in the collectoremitter side and clamp sub circuit is in the collectorgate side. Combination of the dynamic sub circuit and clamp sub circuit minimize the disadvantageous of both sub circuits. Dynamic sub circuit can minimize the oscillation of the voltage during switching times and leads to decrease the switching losses of the clamp subcircuit. Protection of the IGBTs against overvoltage can be done by clamp sub circuit therefore it can avoid using big snubber circuit [4]. The formulas in [4] was derived for three IGBTs in series and the values of the dynamic and the static sub circuits was calculated by them. The clamp circuit can clamp the voltage across fastest IGBT(s) and prevent overvoltage which comes from different delays in the switching times of the IGBTs and deviation in the IGBT parameters. Figure 1 presents three IGBTs in series which is protected by the clamp sub circuit in the gate-collector side and the static and the dynamic sub circuits in the collector-gate side. $R_{\text {Clamp }}$ can limit the current and protect the zener diodes. Diode in series with the zener diodes avoids creating low pass impedance in the clamp string when IGBT is on [5]. Current passing through the gate resistance should pass through the clamp circuit during the switching process. Therefore, the zener diodes should be able to pass the same value of surge reverse current through during this time. Actually, the zener diode with at least surge reverse current equal to the maximum current which is passing through the gate resistance during switching process is needed. In this paper, three series IGBTs are used in a buck converter and tested in different level of voltages. Finally, they are tested by clamp voltage close to the blocking voltage of the IGBT. Maximum 2A current is in the output of the converter. Both switching off and on process are the focus in this paper. Both simulation and experimental results are compared but stray inductances of the circuit and parasitic capacitances of the components have influence on the experiments. Both results of the simulations and the experiments are in the following.

\section{Designed buck converter}

Buck converter which can handle around $3 \mathrm{kV}$ was made by three series IGBTs $(1.2 \mathrm{kV})$. Figure 2 and 3 shows the designed converter in Pspice and laboratory set-up. Semi active gate driver circuit was used in the laboratory design. Three $1.2 \mathrm{kV}$ silicon diodes are used as free-wheeling diodes in the converter structure. The clamp circuit can clamp the IGBTs in around $1 \mathrm{kV}$.

The Lab set-up was designed to be compact and have low inductive circuit. 


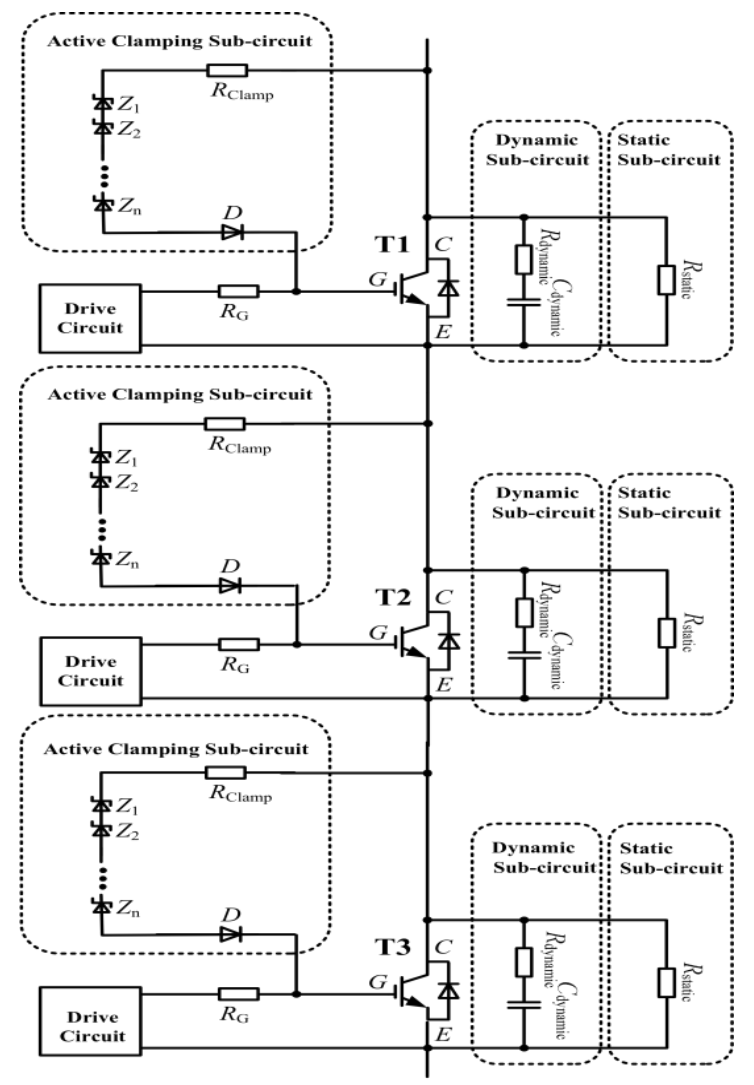

Figure 1. Three IGBTs in series protected by method in reference [1]. Clamp sub circuit in the collectorgate side and dynamic and static sub circuits in the collector-emitter side.

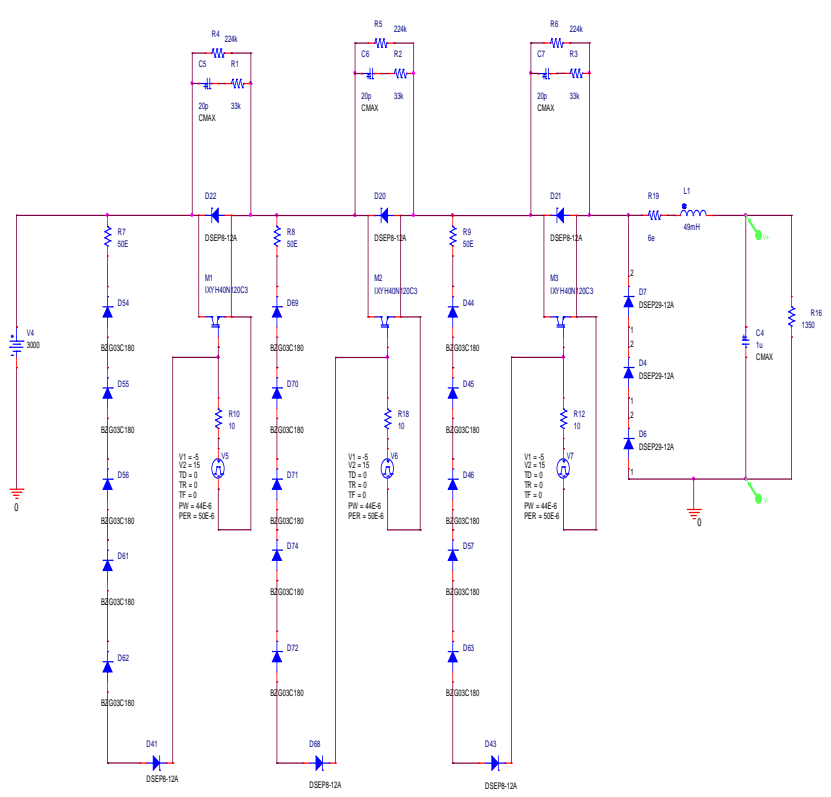

Figure 2. Buck converter circuit in Pspice. Three IGBTs in series leads to converter can handle around $3 \mathrm{kV}$ in input. Clamp sub circuit and static and dynamic sub circuit protect the string of the IGBTs.

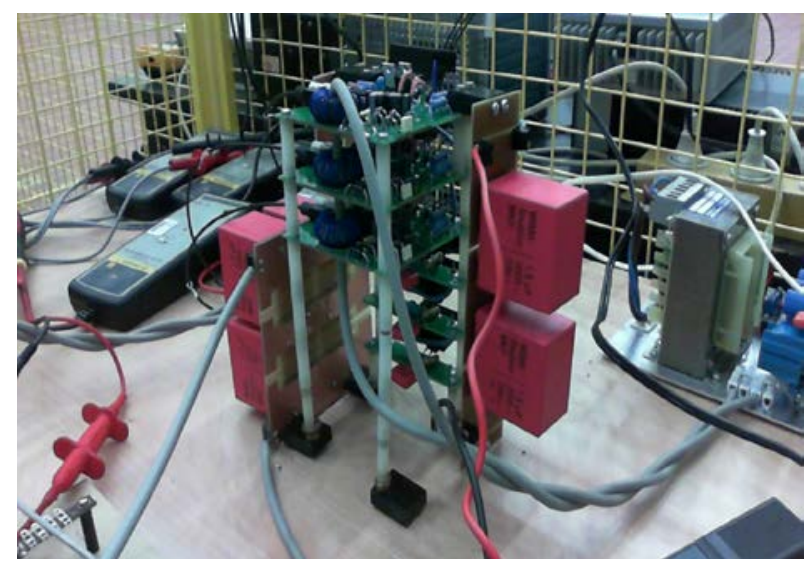

Figure 3. Buck converter set-up with three IGBTs in series in the laboratory. Compact structure leads to have low inductive circuit.

\section{Simulations}

Different delays in the switching times of the IGBTs lead to have transient breakdown voltage across fastest IGBT(s) and slowest IGBT(s) during switching off and on process, respectively. The simulations show the method in [4] can prevent overvoltage across fastest IGBT(s) during switching off process and protect them when there are three IGBTs in series with input voltage $3 \mathrm{kV}$ and clamping voltage $1 \mathrm{kV}$. Figure 4 shows voltages across the IGBTs in the string. One of the IGBTs turns off and on $2 \mu$ s faster than the others. During switching off delay between the IGBTs, clamp circuit of the fastest IGBT acts and protect the IGBT. During switching on delay between the IGBTs, fastest IGBT turn on faster and two other IGBTs should share the voltage during this delay. Therefore, clamp subcircuits of them acts and clamp IGBTs in $1 \mathrm{kV}$.

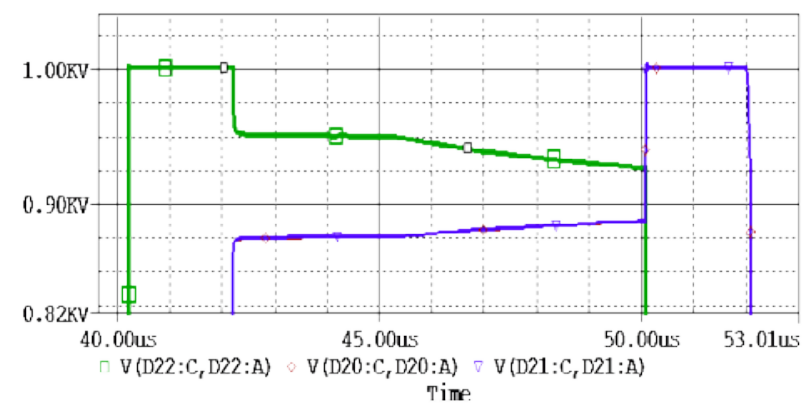

Figure 4. Voltages across IGBTs with $2 \mu s$ delay between fastest IGBT and the others in Pspice.

\section{Experiments}

\subsection{Switching off process}

The non-synchronized switching leads to have overvoltage across at least one IGBT in the series connection of them. Therefore, using the clamp circuit is an easy and reliable way to protect the IGBTs against overvoltage during switching off process. Figure 5 
shows the voltage across the IGBTs during switching off process when the clamping voltage is $200 \mathrm{~V}$. There are delays between IGBTs and it leads to different level of voltage across them during switching off process. Fastest IGBT turns off first and its clamp circuit starts to act and clamp the voltage across the IGBT. Therefore, maximum current passing through the gate resistance during switching off process (1A in this design) starts to go through the clamp circuit string until the other IGBTs turn off. During this time there is a small spike on the voltage curve which is the voltage drop across the clamp resistor. After a while the second fastest IGBT turns off and its clamp circuit starts to act and pass the current of the gate resistance through. Finally, the third IGBT turns off and the IGBTs try to share the voltage. Static resistor forces the IGBTs to have the same voltages by charging and discharging their parasitic capacitances.

Figure 6 and 7 shows the voltages across the IGBTs in clamping voltage around $400 \mathrm{~V}$ and $800 \mathrm{~V}$, respectively, during switching off process. In the both case, there is delays between the switching times of the IGBTs. Fastest IGBT is clamped in the desired voltage and prevented overvoltage. The role of zener diode is very important and it is essential to pay attention to the power loss limitation of the zener diode. If there is big delay between switching time of the IGBTs, the zener diodes have to pass current during larger time. Therefore, their losses increase and may lead to damage them. In addition of losses, the zener diodes should be fast enough compare to the IGBTs to be able to clamp the IGBTs without delay.

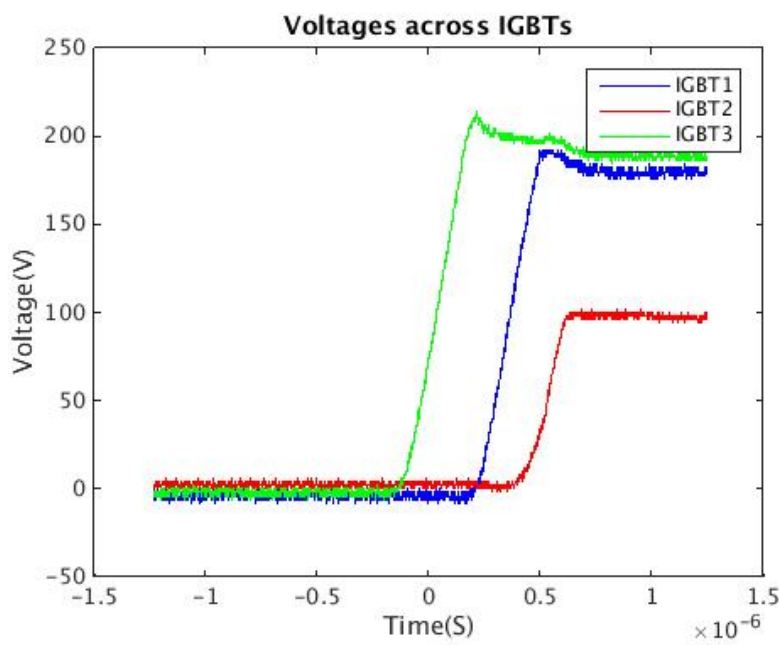

Figure 5. Voltage across the IGBTs in series connection with the clamp voltage $200 \mathrm{~V}$ during switching off process in the Lab set up of the buck converter design. Fastest and second fastest IGBTs have been clamped in $200 \mathrm{~V}$ and the clamp circuits protect them in the desired voltage.

*IGBT3 is the IGBT near to the inductor and the free-wheeling diodes. IGBT1 is the IGBT near to the input.

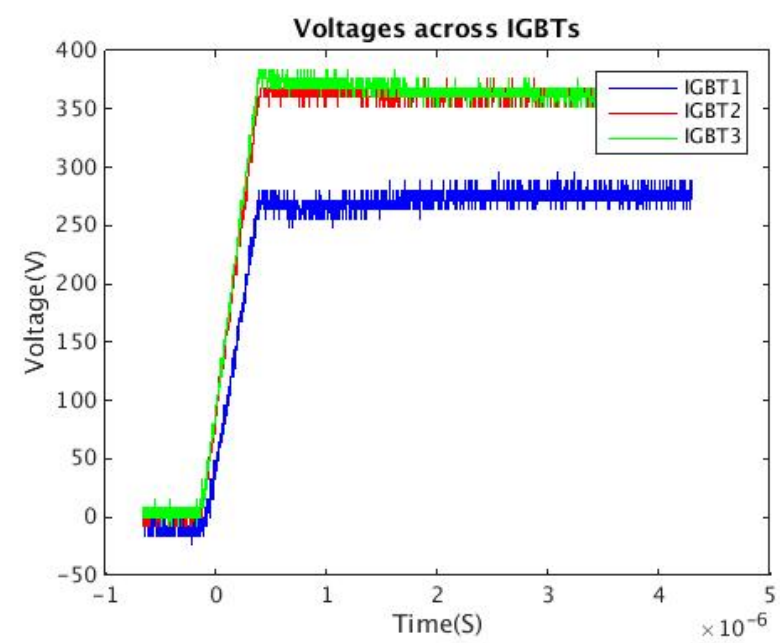

Figure 6. Voltage across the IGBTs in series connection with the clamp voltage $400 \mathrm{~V}$ during switching off process in the Lab set up of the buck converter design. Fastest and second fastest IGBTs have been clamped in $400 \mathrm{~V}$.

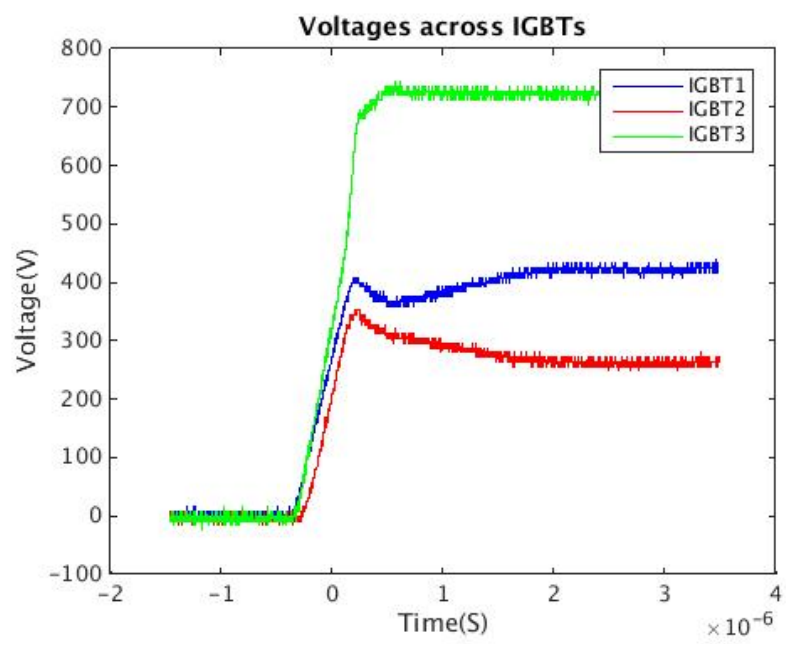

Figure 7. Voltage across the IGBTs in series connection with the clamp voltage $800 \mathrm{~V}$ during switching off process in the Lab set up of the buck converter design. Fastest IGBT has been clamped in $800 \mathrm{~V}$ protected against overvoltage.

\subsection{Switching on process}

Switching on process is as important as switching off process in series connection of IGBTs in a converter design. Similar to the simulations, first fastest IGBT turns on during the switching on process and other IGBTs start to share the voltage during this interval. Therefore, the clamp circuits of these IGBTs start to act and pass current through. Compare to the simulations, the stray inductances and parasitic capacitances of the circuit have influence on the behavior of the converter. Interaction between inductive and capacitive parts in the buck converter structure leads to high frequency oscillations on the voltages of the IGBTs and create transient breakdown. Figure 8, 9 and 10 show voltages across the three IGBTs in different switching delays 
positions when the clamp voltage is $200 \mathrm{~V}$. The voltage across the IGBT module which is nearest to the inductor and the free-wheeling diodes is the green curve. High frequency oscillations is on the voltage curve when this IGBT has the second and third fastest switching delays. Actually, interaction between inductor and parasitic capacitances of the free-wheeling diodes and this IGBT leads to create transients. The transients is dangerous for the system in higher voltages and a solution should be considered. The conducting loss of this IGBT can damp the oscillations when it is the fastest to turn on during switching on process, see figure 10 . Therefore, as a solution, always nearest IGBT to the free-wheeling diodes and the inductor should be fastest to turn on during the switching on process in a converter design. Actually, pre-adjustment of the switching delays is needed.

Figure 11 and 12 shows the voltages across the IGBTs during switching on process at clamping voltage $400 \mathrm{~V}$ and $800 \mathrm{~V}$, respectively. The switching on process is normal in all levels of voltages when pre-adjustment is considered. In addition, figure 11 shows the process of sharing the voltage between the second and the third fastest IGBTs during the switching on process after turning on of the fastest IGBT. These two IGBTs try to share the voltage but the input voltage is not big enough to their clamp circuits start to act. After a while these IGBTs turn on and the voltages drop.

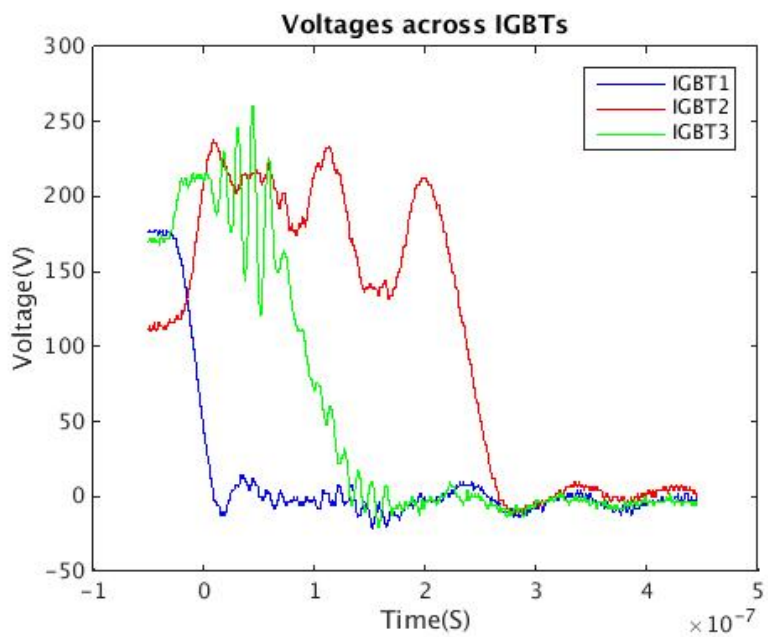

Figure 8. Voltages across the IGBTs in series connection during the switching on process when the clamp voltage is $200 \mathrm{~V}$. At this time, IGBT3 is the second fastest IGBT during the switching on process. High frequency oscillations create because of the interaction between the inductor and parasitic capacitances of the diodes and IGBT3.

*IGBT3 is the IGBT near to the inductor and the free-wheeling diodes. IGBT1 is the IGBT near to the input.

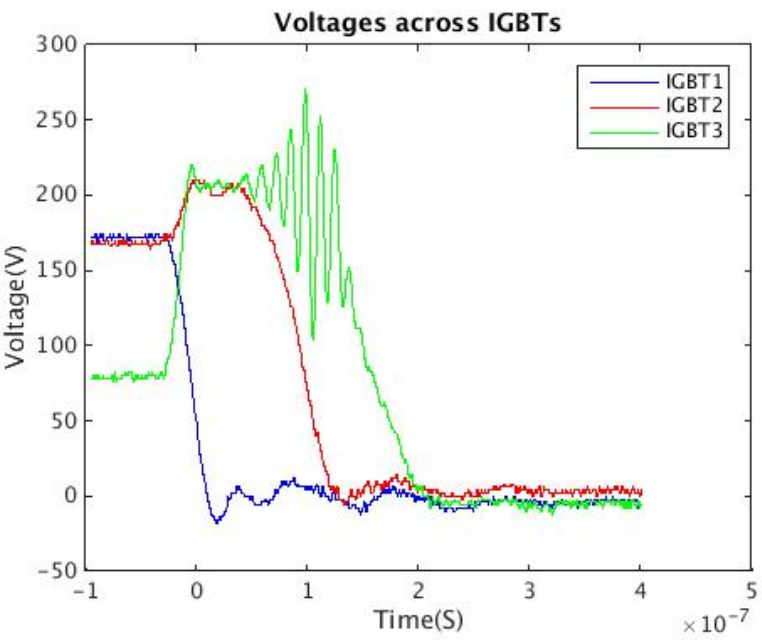

Figure 9. Voltages across the IGBTs in series connection during the switching on process when the clamp voltage is $200 \mathrm{~V}$. At this time, IGBT3 is the slowest IGBT during the switching on process. Still there is high frequency oscillations on the voltage of IGBT3.

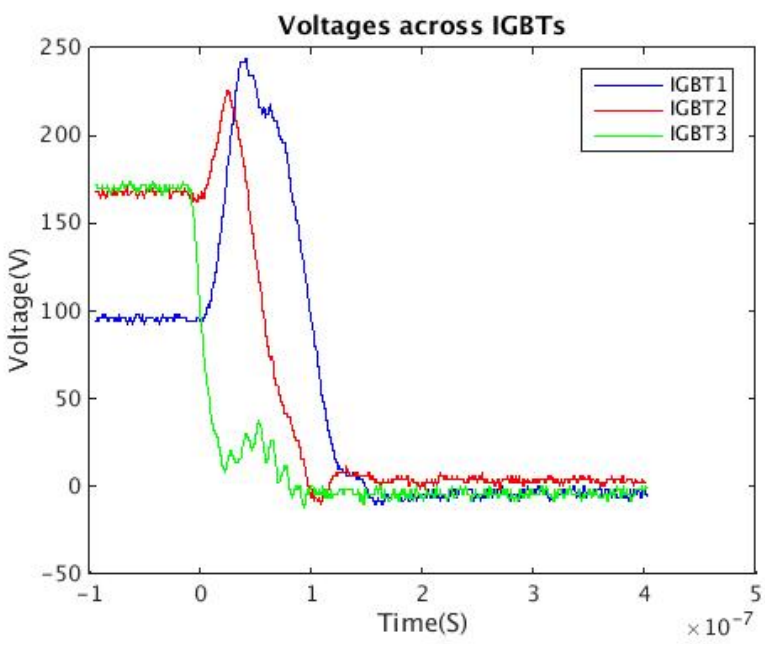

Figure 10. Voltages across the IGBTs in series connection during the switching on process when the clamp voltage is $200 \mathrm{~V}$. At this time, IGBT3 is the fastest IGBT during the switching on process and its conducting loss damps the oscillations. As a solution, IGBT which is nearest to the inductor and freewheeling diode(s) should always be the fastest one during the switching on process. 


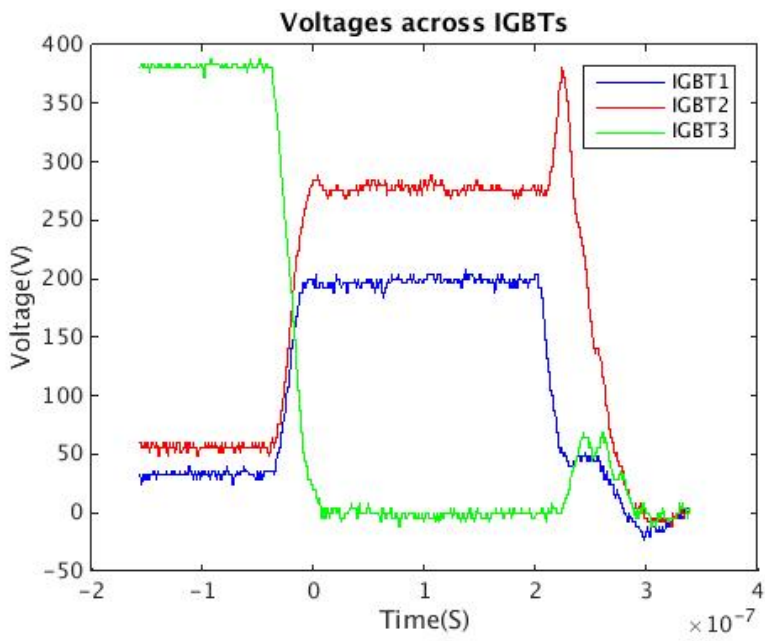

Figure 11. Voltages across the IGBTs in series connection during the switching on process when the clamp voltage is $400 \mathrm{~V}$. IGBT3 is the fastest IGBT during the switching on process. This figure shows the voltage sharing between the other IGBTs after turning on of the fastest one.

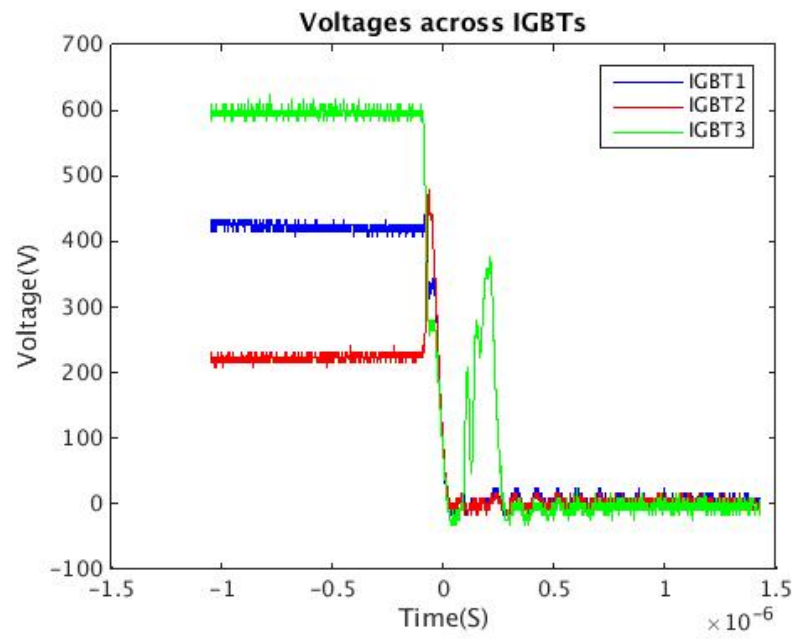

Figure 12. Voltages across the IGBTs in series connection during the switching on process when the clamp voltage is $800 \mathrm{~V}$. IGBT3 is the fastest IGBT during the switching on process.

\section{Conclusion}

In this paper, experimental test of the high voltage converter with the three IGBTs in series which is protected by the method in [4], shows high voltage converter can be made in different levels of voltages with several dozens of IGBTs.

Pre-adjustment of the switching delays can protect IGBTs against transient breakdown during switching on process.

In addition, power loss and speed of zener diodes are significant factors to be considered to have fast clamp circuit which is able to protect IGBTs against transient breakdown from the beginning of switching off process and handle longer switching delays during this time.

\section{References}

[1] Ting Lu, Zhengming Zhao, Shiqi Ji, Hualong Yu, Liqiang Yuan, Fanbo He, Yingchao Zhang, Fei Kong, Design of Voltage Balncing Control Circuit for Series Connected HV-IGBTs, 2013 International Conference on Electrical Machines and Systems, Oct. 26-29, 2013, Busan, Korea.

[2] Tonny W. Rasmussen, “Active gate driver for $\mathrm{dv} / \mathrm{dt}$ control and active voltage clamping in and IGBT stack”, EPE2005 Dresten.

[3] Robin Voigt, Karsten Handt, Melanie Eckert, "Fully digitized quasi-continuous working gatedrive unit for 1200 V-IGBTs”.

[4] Ting Lu, Zhengming Zhao, Shiqi Ji, Hualong Yu, Liqiang Yuan, Parameter Design of Voltage Balancing circuit for series connected HV-IGBTs, 2012 IEEE $7^{\text {th }}$ international power electronics and motion power conference-ECCE Asia.

[5] Ruchira Withanage, Noel Shammas, Series connection of Insulated Gate Bipolar Transistors (IGBTs), IEEE TRANSACTIONS ON POWER ELECTRONICS,VOL.27, NO 4, April 2012. 